\title{
Genetic mapping and manipulation: Chapter 10-Suppressor mutations*
}

David Fay ${ }^{\S}$, Department of Molecular Biology, University of Wyoming, Laramie, Wyoming 82071-3944 USA

Wade Johnson $\$$, Howard Hughes Medical Institute, Department of Molecular, Cellular, and Developmental Biology, University of Colorado, Boulder, CO 80309 USA

\section{Table of Contents}

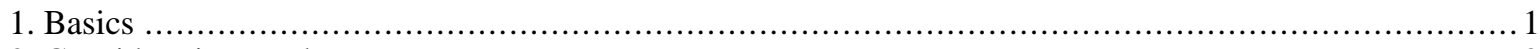

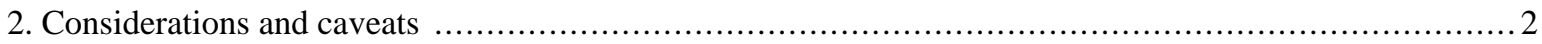

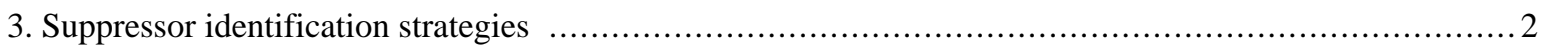

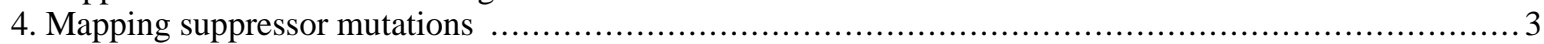

\section{Basics}

Suppressor and enhancer screens are powerful ways to uncover more information about a known gene or mutation, including the identification of other interacting components in a pathway. Very simply, a suppressor screen starts with a known mutation and then identifies second-site mutations that suppress the mutant phenotype. Enhancer screens are just the opposite and are discussed in the above section along with synthetic mutations. One classic example is the let-60 (gf) suppressor screen carried out in recent years by the Han lab. In this screen, let-60 worms were mutagenized by EMS and scored for suppression of the let-60 multivulval (Muv) mutant phenotype. Suppression of this phenotype leads to either a normal vulva or loss of the vulva, vulvalless (Vul). From this screen a variety of genes were isolated and characterized. Many of these genes (or at least the alleles isolated) possess silent phenotypes on their own and hence would only have been isolated by such a screen. This is one of the greatest strengths of a suppressor screen. Also, all of the genes so far characterized from this screen have led to a greater understanding of the ras pathway not only in worms but in other organisms as well.

*Edited by Victor Ambros. Last revised May 6, 2005. Published February 17, 2006. This chapter should be cited as: Fay, D. and Johnson, W. Genetic mapping and manipulation: Chapter 10-Suppressor mutations (February 17, 2006), WormBook, ed. The C. elegans Research Community, WormBook, doi/10.1895/wormbook.1.99.1, http://www.wormbook.org.

Copyright: ( $) 2006$ David Fay and Wade Johnson. This is an open-access article distributed under the terms of the Creative Commons Attribution License, which permits unrestricted use, distribution, and reproduction in any medium, provided the original author and source are credited.

${ }^{\S}$ To whom correspondence should be addressed. E-mail: davidfay@uwyo.edu or wade.johnson@ colorado.edu 


\section{Considerations and caveats}

Before starting a suppressor screen, you obviously must have a mutation to suppress. The better characterized the mutation, the better able you will be to design an effective screen, as the characteristics of your mutation will affect the types of suppressors you can hope to isolate. As a general rule, it is almost always easier to isolate suppressors of weak or partial LOF mutants than for strong LOF or null mutants. The reason is that some suppressors are likely to be mutations in negative regulators of your gene, possibly at the level of transcription, protein stability, or protein activity. In the case of null mutations where your protein has either no activity or isn't being made, such suppressors will be ineffective, as they have nothing to work with. For example, if your mutation is a partial loss of function with $20 \%$ normal activity, a suppressor that increases the abundance or activity of your protein by five-fold will theoretically bring you back to normal levels. The same cannot be said if your protein has $0 \%$ activity.

The suppressors you obtain will potentially fall into two classes: informational suppressors and functional suppressors. The former class includes gene products that will suppress your mutation through a generic mechanism, such as the suppression of a stop codon. Other informational suppressors include mRNA degradation mutants (smg genes) and protein-degradation mutants (ubiquitination enzymes). For the most part, this class of suppressors is less interesting but often cannot be avoided. Knowing the molecular lesion within your mutant of interest will allow you to determine what types of informational suppressors you might expect to uncover within your screen. The more relevant class of functional suppressors acts through mechanisms that will hopefully shed light on the process or gene of interest.

The question then becomes how can one determine which suppressor mutations are informational and which are functional? By setting up a series of basic experiments designed to test the characteristics of the individual suppressor mutations, one can easily avoid wasting inordinate amounts of time on suppressors that are non-specific. A variety of experiments could be designed to answer this question; a few are listed below. These are not necessarily the only experiments that could be done, but they are a good place to start. Each mutant being suppressed will lead to unique experiments that can be carried out.

First: Cross suppressors to multiple mutant alleles of the gene of interest and assay for suppression. In addition, if RNAi produces a phenotype for your mutation, this may provide a complementary approach. Although this may provide a good test for overall suppression, keep in mind that suppressors that are not effective on other alleles may still be interesting (i.e., functional) because of their allele specificity. This test must be measured against the other tests to determine the validity of the suppressors.

Second: Use RNAi to disrupt any residual message from the mutant of interest in the mutant:suppressor background. If the suppressors are functional and not informational, you may (not) see a reversion back to the 100\% mutant phenotype. However, the same stipulation stated above for alternative alleles also holds true for RNAi. Moreover, certain informational suppressors may act by affecting mRNA degradation, and thus could compromise the RNAi pathway. This can potentially complicate interpretation of tests using RNAi.

Third: In the case of a mutation of interest containing a premature stop codon, cross the suppressor into a strain containing another mutant gene with the same premature stop codon (e.g., an opal stop in both cases). Suppression of this unrelated mutated gene suggests you have isolated an informational suppressor.

Fourth: If the gene of interest is within a known biochemical pathway, test other members of this pathway, either by using previously identified mutations or RNAi. These experiments should reveal the extent of suppression as being either specific to your gene of interest or encompassing the entire pathway.

\section{Suppressor identification strategies}

Your specific suppressor screen will obviously depend on many factors, such as whether or not the mutation is viable or easy to score by its plate phenotype. In the case of embryonic or larval lethal phenotypes, it is useful first to link your mutation $(\boldsymbol{m})$ to a nearby and easily recognizable morphological marker (such as $\boldsymbol{d p y}$ ). The $\boldsymbol{d p y} \boldsymbol{m}$ chromosome is then preferably balanced by a regional deficiency or chromosomal rearrangement that discourages recombination or by a set of closely spaced morphological markers. The idea is that you want a well-balanced strain where you never see viable Dpy animals (or can easily tell if a rare recombination event has taken place). The strain is then mutagenized, and plates are screened in subsequent generations for the presence of viable Dpy animals. 
An alternative approach (if your mutation is cloned) would be to rescue your mutant using an extrachromosomal array that contains a wild-type copy of the gene as well a marker, such as GFP. One could then mutagenize this resuced strain and look for non-green (array-minus) animals that don't show the phenotype. This may be particularly useful for lethal or other easily scorable mutations. As an added option, you could use a worm sorter to do much of the work for you (separating any green from non-green animals), making this a very powerful approach.

A more modern method for finding suppressors may be through the use of RNAi feeding. Namely, plowing through the "complete" genome library and looking for revertants. This approach has the major added advantage that the molecular identity of the suppressing clones are immediately known, in contrast to the time consuming procedure of cloning mutations. However, it is important to recall that RNAi feeding is not a terribly effective means for inactivating genes, and you may miss a large number of potential suppressors. Also, it may be prudent to first attempt a reasonably sized genetic suppressor screen first, to determine whether your mutation or allele is likely to be suppressable. If you seem to be getting reasonable numbers of suppressors, then investing in an RNAi screen may be more attractive.

\section{Mapping suppressor mutations}

Another key consideration in suppressor mapping is the creation of mapping strains. Depending on the suppressors isolated, many suppressors will be silent (i.e., they will have no observable phenotype on their own). Therefore, to map these silent suppressors the original suppressed mutation must be included in all your mapping strains. Another consideration is mating difficulties; some mutations may affect male fertility in the homozygous state. If this is the case, then it is always best to mate males into the mapping strain rather than into the suppressed strain to obtain heterozygote fertile males. The reason for this is to reduce the risk of losing the suppressor. Figure 1 shows a typical mating between a specific suppressor and a mapping strain.

$$
\begin{array}{ll}
\frac{+}{d p y \text { unc }}+\frac{+}{m} \bigcirc & \frac{\sup }{\sup } \frac{m}{m} \uparrow \\
& \begin{array}{l}
\text { Pick M worms from plates that also } \\
\text { throw Dpy Unc worms }
\end{array}
\end{array}
$$

sup on dpy unc chromosome

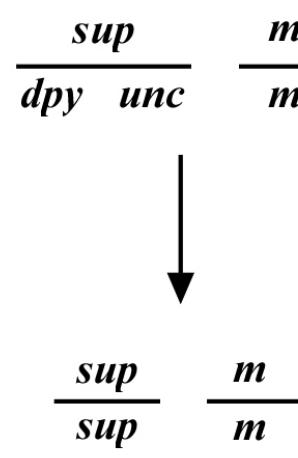

\section{sup not on dpy unc chromosome}

$$
\frac{\sup }{+} \frac{+\quad+}{d p y \text { unc }} \frac{m}{m}
$$

Step 2

\section{Pick Sup worms}

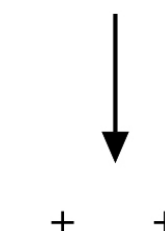

$$
\frac{\text { sup }}{\text { sup }} \frac{+\quad+}{d p y \text { unc }} \frac{m}{m}(2 / 3)
$$

Step 3

\section{AND}

$$
\frac{\sup }{\sup } \quad \frac{m}{m}(1 / 3)
$$

Figure 1. 
The worms isolated in step 3 are then scored for the Dpy and Unc phenotypes in their progeny. If the suppressor is on the same chromosome as the markers and is very close, then $\sim 100 \%$ of the worms will not throw the markers (i.e., their progeny will not express these phenotypes). If the suppressor is not on the same chromosome, then two-thirds of the worms should throw the markers (see Two-point mapping with genetic markers for a better explanation). These numbers, of course, are to be expected if the world were perfect, which it is not. Recombination makes this a little more difficult and a little more telling.

Recombination at this step may also supply vital information for suppressors that are on the same chromosome as the markers. Because of recombination, a few of the progeny from step 3 may be either Dpy or Unc. The frequency with which these recombination events arise allows you to map the suppressor to a specific chromosome and provides information about the suppressor's direction and distance from the known markers. Three-point mapping is then carried out with other markers that should bookend the mutation. For a further and more thorough explanation, please see the Two-point mapping with genetic markers and Three-point mapping with genetic markers sections.

All WormBook content, except where otherwise noted, is licensed under a Creative Commons Attribution License. 